\title{
Imaging assessment after SBRT for hepatocellular carcinoma
}

\author{
Connie Yip ${ }^{1}$, Tiffany Priyanthi Hennedige², Gary J. R. Cook ${ }^{3}$, Vicky Goh ${ }^{3}$ \\ 'Radiation Oncology, National Cancer Centre Singapore, Singapore 169610, Singapore. \\ ${ }^{2}$ Oncologic Imaging, National Cancer Centre Singapore, Singapore 169610, Singapore. \\ ${ }^{3}$ Cancer Imaging, King's College London, London WC2R 2LS, United Kingdom.
}

Correspondence to: Dr. Connie Yip, Radiation Oncology, National Cancer Centre Singapore, Singapore 169610, Singapore. E-mail: connie.yip.s.p@singhealth.com.sg

How to cite this article: Yip C, Hennedige TP, Cook GJR, Goh V. Imaging assessment after SBRT for hepatocellular carcinoma. Hepatoma Res 2020;6:44. http://dx.doi.org/10.20517/2394-5079.2020.30

Received: 24 Mar 2020 First Decision: 25 May 2020 Revised: 25 May 2020 Accepted: 26 May 2020 Published: 10 Jul 2020

Academic Editor: Su Pin Choo Copy Editor: Cai-Hong Wang Production Editor: Tian Zhang

\begin{abstract}
The use of stereotactic body radiotherapy (SBRT) in hepatocellular carcinoma (HCC) has increased over the past few decades. Thus, accurate evaluation of post-SBRT treatment response is essential to avoid over-treatment of responders as well as missing the opportunity to salvage non-responders. There are some intricate imaging differences after liver SBRT compared to those observed after conventional fractionated radiotherapy and other locoregional treatment. We aim to review the imaging changes that occur following SBRT for HCC and their potential clinical implications.
\end{abstract}

Keywords: Imaging, liver, stereotactic body radiotherapy, hepatocellular carcinoma

\section{INTRODUCTION}

Radiation therapy for liver malignancies has evolved over the past few decades. In the past, radiation was predominantly used as a palliative modality due to the limited tolerance of whole liver irradiation. However, with the technological advances achieved with improved resolution of on-board imaging and the ability to deliver highly conformal radiotherapy, we were then able to irradiate liver tumours with high precision and limited bystander damage to normal tissues. Stereotactic body radiotherapy (SBRT) is characterised by high dose per fraction, typically in the range of 5-25 Gy over 1-10 fractions, which is enabled by accurate tumour localisation using daily image guidance. The adoption of SBRT has increased exponentially in primary and secondary liver malignancies with promising local control rates and favourable toxicity profiles ${ }^{[1-5]}$. SBRT has also been shown to be a useful local therapy to bridge patients awaiting transplant in primary hepatocellular carcinoma $(\mathrm{HCC})^{[6]}$. 
Table 1. The different imaging treatment response evaluation criteria for HCC

\begin{tabular}{|c|c|c|c|}
\hline Treatment response & RECIST 1.1 & MRECIST & EASL \\
\hline Tumour measurements & Uni-dimensional of target lesions & $\begin{array}{l}\text { Uni-dimensional of viable tumours } \\
\text { (arterial phase enhancement) }\end{array}$ & $\begin{array}{l}\text { Bi-dimensional of viable tumours } \\
\text { (arterial phase enhancement) }\end{array}$ \\
\hline Number of lesions & 2 per organ & 2 per organ & Not specified \\
\hline Complete response (CR) & Disappearance of all target lesions & $\begin{array}{l}\text { Disappearance of any intratumoral } \\
\text { arterial enhancement in all target } \\
\text { lesions }\end{array}$ & $\begin{array}{l}\text { Disappearance of any intratumoral } \\
\text { arterial enhancement in all target } \\
\text { lesions }\end{array}$ \\
\hline Partial response (PR) & $\begin{array}{l}\geq 30 \% \text { reduction in sum of longest } \\
\text { diameters of target lesions }\end{array}$ & $\begin{array}{l}\geq 30 \% \text { reduction in sum of longest } \\
\text { diameters of viable target lesions }\end{array}$ & $\begin{array}{l}\geq 50 \% \text { reduction in sum of the product } \\
\text { of bi-dimensional diameters of viable } \\
\text { target lesions }\end{array}$ \\
\hline Progressive disease (PD) & $\begin{array}{l}\geq 20 \% \text { increase in sum of longest } \\
\text { diameters of target lesions }\end{array}$ & $\begin{array}{l}\geq 20 \% \text { increase in sum of longest } \\
\text { diameters of viable target lesions }\end{array}$ & $\begin{array}{l}\geq 25 \% \text { increase in sum of the product } \\
\text { of bi-dimensional diameters of viable } \\
\text { target lesions }\end{array}$ \\
\hline Stable disease (SD) & Does not meet PR or PD & Does not meet PR or PD & Does not meet PR or PD \\
\hline
\end{tabular}

HCC: Hepatocellular carcinoma

Nonetheless, treatment response assessment after high ablative doses of radiation can be challenging due to the different radiation dose deposition profiles compared to conventional fractionated radiotherapy. Here, we aim to review the imaging characteristics associated with SBRT in HCC and their implications in our clinical practice.

\section{HISTOPATHOLOGICAL CHANGES AFTER RADIATION}

During the acute phase, which is typically defined as less than 3-4 months after radiation, there is deposition of fibrin and subsequently collagen within the centrilobular venules causing obliteration of these small vessels with relative sparing of the larger veins and arterioles. This causes reactive hyperaemia and also hepatic cell loss within the liver ${ }^{[7,8]}$. This is collectively known as veno-occlusive disease. In the chronic phase, there is significant reduction of hyperaemia with some degree of hyperplasia and parenchymal fibrosis ${ }^{[8]}$.

\section{IMAGING RESPONSE ASSESSMENT CRITERIA}

Although RECIST 1.1 is a universally accepted set of radiological response evaluation criteria in solid tumours $^{[9]}$, these criteria do not apply well in HCC as they are based solely on uni-dimensional tumour measurements. Several other response evaluation criteria, which are more sensitive for HCC, have been proposed, including the EASL and modified RECIST (mRECIST) criteria [Table 1] ${ }^{[10,11]}$. The EASL criteria use bi-dimensional measurements of viable enhancing tumours, whereas mRECIST uses uni-dimensional measurement of viable tumours. There are a few studies that compared the different evaluation criteria after SBRT treatment for HCC but no definite conclusions could be drawn regarding the most optimal criteria to use in clinical practice ${ }^{[12,13]}$. Of interest, one study correlated radiological response to pathological response in 38 patients who underwent orthotopic liver transplants for $\mathrm{HCC}^{[14]}$. The radiological criteria used included EASL, RECIST and mRECIST. All radiological response criteria compared poorly against the actual pathological response. The positive predictive values and negative predictive values were $73 \% / 29 \%$ (EASL), $71 \% / 32 \%$ (RECIST) and $74 \% / 40 \%$ (mRECIST), respectively. Both computed tomography (CT) agreement (22\%-39\%) and magnetic resonance imaging (MRI) agreement (31\%-39\%) with pathologic findings were poor, irrespective of the imaging criteria used. This highlighted the difficulty of using imaging to predict pathological treatment response after SBRT.

\section{EARLY IMAGING CHANGES}

Similar to the histopathological changes described above, corresponding imaging changes can be observed during the acute post-SBRT phase. One important point to remember is that the conventional well-defined radiation field edges observed with the use of two- or three-dimensional radiation techniques are no longer seen in SBRT treatment, which uses multiple, often non-coplanar, radiation fields. 


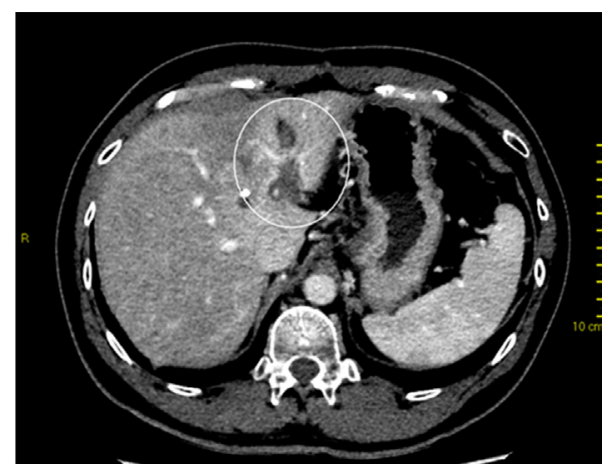

Figure 1. Contrast-enhanced computed tomography at 3 months after stereotactic body radiotherapy showing reactive hyperaemia in the surrounding irradiated non-tumourous liver parenchyma

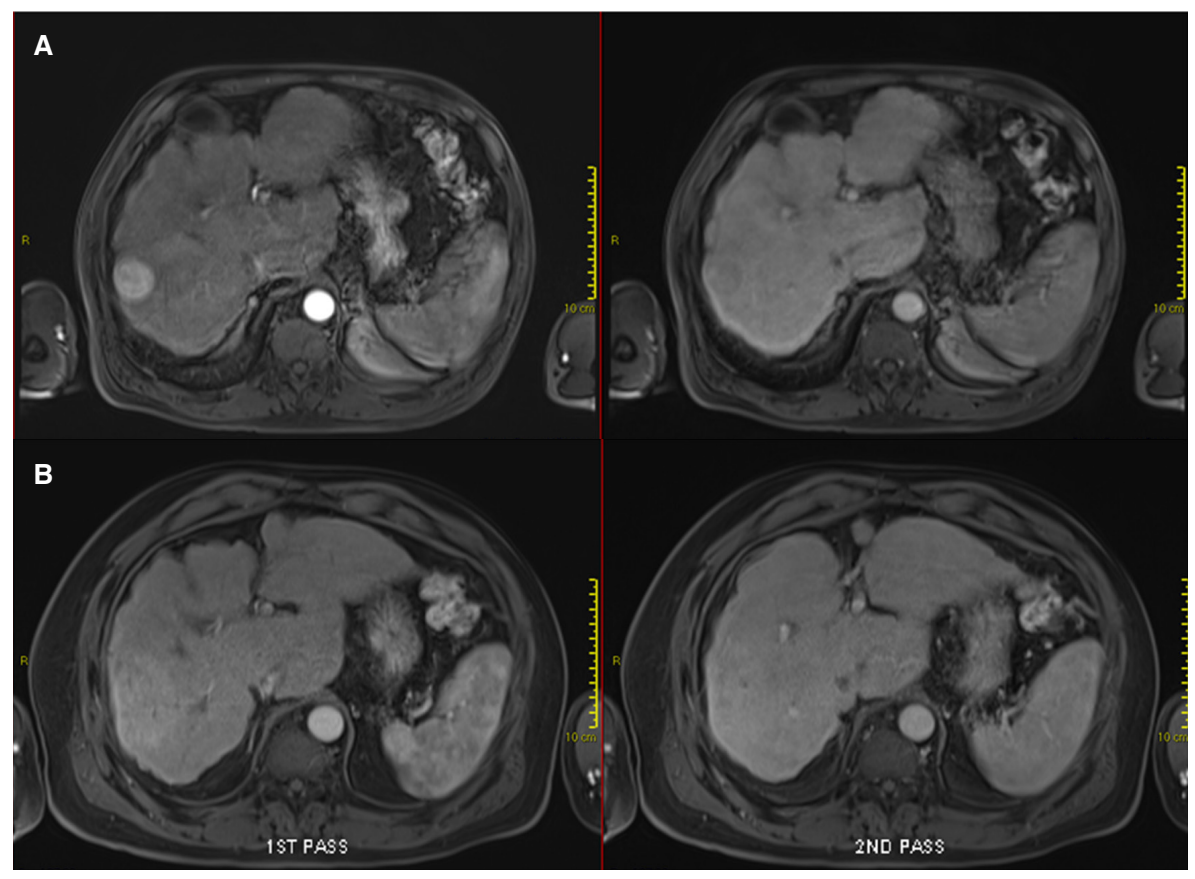

Figure 2. Contrast-enhanced magnetic resonance imaging demonstrating early imaging changes before and after stereotactic body radiotherapy (SBRT): pre-SBRT showing typical arterially enhancing lesion with subsequent wash-out (A); and 3 months after SBRT showing the surrounding arterial enhancement without subsequent wash-out observed, indicative of reactive hyperaemia (B)

During the very early phase, hepatic oedema occurs due to decreased venous outflow secondary to centrilobular obstruction. This is manifested as hypoattenuation on unenhanced CT and hyper-attenuation on contrast-enhanced $\mathrm{CT}^{[15]}$. On MRI, this is manifested as low signal on T1-weighted images with high signal on T2-weighted images. On diffusion-weighted imaging (DWI), there may be mild restricted diffusion and a slight increase in apparent diffusion coefficient (ADC). Periportal oedema can also occur, although this is short-lived and not specific to radiation therapy. On MRI, this can be observed as widening of periportal space with mild high signal intensity on T2-weighted images ${ }^{[16]}$.

Following that, reactive hyperaemia is often seen in the surrounding irradiated non-tumourous liver parenchyma as early as 1 month after treatment and peaks at approximately 3 months ${ }^{[17,18]}$ [Figures 1 and 2]. This focal liver reaction could be difficult to differentiate from residual tumour, a phenomenon also known as "pseudoprogression". However, reactive arterial hypervascularity is usually followed by no delayed phase washout as opposed to viable tumours ${ }^{[17]}$. This is a useful tip during SBRT response assessment to avoid 
misdiagnosing this reactive feature as tumour progression. Focal steatosis may also occur, which is typified by decreased attenuation on CT and signal drop on opposed-phase $\mathrm{MRI}^{[16]}$. In rare circumstances, temporary biliary dilatation is observed, which tends to resolve after a few months ${ }^{[16]}$.

\section{LATE IMAGING CHANGES}

Months after SBRT, the injured hepatocytes are gradually replaced by fibrosis. CT is not usually sensitive in detecting liver fibrosis, although perfusion CT may be more useful for this purpose ${ }^{[19]}$. This chronic effect can be better appreciated on MRI with low signal on T1- and T2-weighted images. During the earlier stages of fibrosis, moderate arterial enhancement is observed, which persists in the delayed phase. As this progresses, arterial enhancement diminishes but progressive enhancement in the delayed phase persists ${ }^{[16]}$.

In most cases, loss of liver volume is observed in the initial 3 months after SBRT. This is followed by subsequent regeneration and increase in liver volume. However, liver atrophy may also occur in some cases with capsular retraction ${ }^{[16]}$ [Figure 3]. In contrast to the early focal steatosis observed, focal sparing of fatty liver (e.g., in pre-existing fatty liver or post-chemotherapy liver changes) may occur in the months following SBRT due to loss of fat in the hepatocytes ${ }^{[16]}$.

Finally, tumour response after SBRT can be challenging, as described above. Tumour shrinkage may not happen in the immediate few months after SBRT. Nonetheless, even in the absence of volumetric reduction, progressive reduction in tumour enhancement is consistent with treatment response. This is typically accompanied by a corresponding increase in $\mathrm{ADC}^{[16]}$. An increment in ADC of $20 \%-25 \%$ was found to be an indicator of SBRT response in a few small retrospective studies ${ }^{[20,21]}$ [Figure 4]. However, the authors did not use pathological response as the study end-point, and hence are subjected to the same challenges as described with the use of radiological response criteria as an end-point. Furthermore, there is as yet no standardisation of DWI acquisition and interpretation, which hinders its use as a standard response evaluation criterion.

\section{ROLE OF POSITRON EMISSION TOMOGRAPHY}

${ }^{18}$ F-Fluorodeoxygluocose positron emission tomography $\left({ }^{18} \mathrm{~F}\right.$-FDG PET) is not recommended in the staging of early HCC due to its low sensitivity in detecting low-grade, well-differentiated HCC against the background liver activity, precluding an accurate primary tumour assessment. Nonetheless, it is more sensitive in detecting extrahepatic disease compared to local tumour staging ${ }^{[2]}$. To our knowledge, there is no study that evaluated the role of ${ }^{18}$ F-FDG PET in treatment response assessment after SBRT for primary HCC. However, baseline ${ }^{18}$ F-FDG PET maximum SUV value $\left(\mathrm{SUV}_{\max }\right)>3.2$ was found to be associated with higher risk of local failure in a cohort of HCC patients treated with $\mathrm{SBRT}^{[23]}$.

Other radionuclides being evaluated in HCC include ${ }^{18} \mathrm{~F}$-Fluorocholine $\left({ }^{18} \mathrm{~F}-\mathrm{FCH}\right)$ that is a biomarker for phosphocholine, which is a major metabolite in cancer cells, and ${ }^{11} \mathrm{C}$-acetat $\left[{ }^{[24]} \cdot{ }^{18} \mathrm{~F}-\mathrm{FCH}\right.$ is reported to have a high sensitivity, approaching $90 \%$, in detecting $\mathrm{HCC}^{[25]}$. A decrease of ${ }^{18} \mathrm{~F}-\mathrm{FCH} \mathrm{SUV} \mathrm{V}_{\max }>45 \%$ was shown to be associated with longer progression-free survival and improved mRECIST response in patients treated with various locoregional therapies, including SBRT for $\mathrm{HCC}^{[26]}$.

The availability of PET/MR imaging is slowly increasing in some parts of the world. This could be a promising tool in HCC combining the superior MRI soft tissue and primary tumour definition, functional aspects of MRI such as DWI and the superior sensitivity of PET in extrahepatic staging.

\section{PREDICTION OF LIVER TOXICITIES}

Although SBRT is relatively well tolerated in most patients, the risk of radiation-induced liver disease (RILD) cannot be underestimated in this group of patients who have pre-existing cirrhosis, particularly those with 


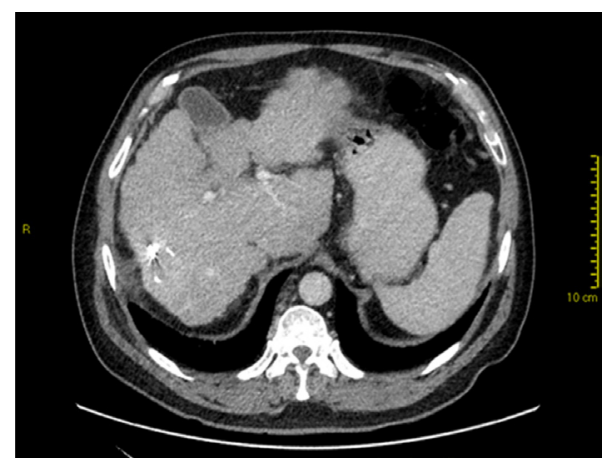

Figure 3. Contrast-enhanced computed tomography at 1 year after stereotactic body radiotherapy showing volume loss with capsular retraction (note fiduciary clips in-situ)

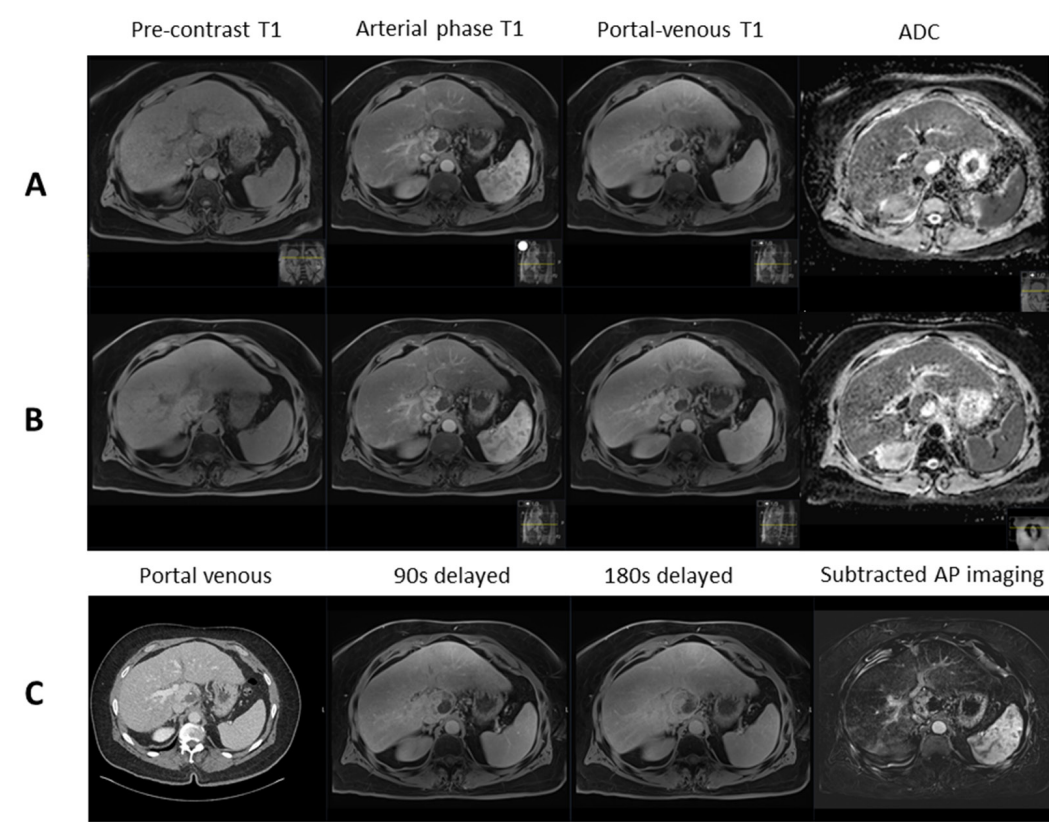

Figure 4. Contrast-enhanced magnetic resonance imaging demonstrating: pre-treatment caudate lobe hepatocellular carcinoma with restricted diffusion ( $\left.A D C 1101 \times 10^{-6}\right)(A)$; post-stereotactic body radiotherapy scan showing no treatment response with restricted diffusion (ADC $\left.917 \times 10^{-6}\right)(B)$; and corresponding contrast-enhanced computed tomography and delayed imaging with hepatobiliary contrast agent showing perfusional changes around the tumour with no response (C). ADC: apparent diffusion coefficient

Child-Pugh B liver dysfunction. Classical RILD, which is characterised by weight gain, hepatomegaly, anicteric ascites and isolated elevation of alkaline phosphatase (ALP) out of proportion to other liver enzymes, is not usually seen following liver SBRT. In contrast, non-classical RILD, which is characterised by markedly elevated transaminases and jaundice, is more commonly seen after partial liver irradiation such as $\mathrm{SBRT}^{[27]}$.

A combination of clinical and/or biochemical criteria such as Child-Pugh and Model for End-stage Liver Disease (MELD) scores are routinely used as a measure of the severity of cirrhosis in the clinical setting. In addition, serum indocyanine green (ICG) clearance is also widely used to evaluate liver function in clinical practice, although it is a direct measurement of neither the hepatic synthetic nor conjugative function ${ }^{[28]}$. ${ }^{99 m}$ Tc-Sulphur Colloid single-photon emission computed tomography $\left({ }^{99 m} \mathrm{Tc}-\mathrm{SC}\right.$ SPECT) is considered a liver function imaging tool. ${ }^{99 \mathrm{~m}} \mathrm{Tc}-\mathrm{SC}$ is taken by Kupffer cells which have similar perfusion as hepatocytes, thus making it a surrogate of perfused hepatocytes ${ }^{[29]}$. Quantitative ${ }^{99 m}$ Tc-SC SPECT uptake was found to have 
significant correlations with ICG retention at $15 \mathrm{~min}$ (ICG-R15) $(r=-0.84, P<0.0001)^{[29]}$ and clinical ChildPugh status ${ }^{[30]}$.

Hepatocyte-specific MRI contrast agents, such as gadoxetate acid (Gd-EOB DTPA), also have the potential to provide information on liver function besides its role as a diagnostic tool in $\mathrm{HCC}^{[31,32]}$. The rate of T1 relaxation time reduction and T1 map quantifications have been shown to correlate well with ICG clearance ${ }^{[31-33]}$. However, its use in this setting requires additional research and validation.

We anticipate that functional liver imaging will play a complementary role to existing cirrhosis severity evaluation criteria, particularly in providing valuable spatial information which can be used to guide HCC treatment. One exciting area of research is the prospect of sparing functional liver as depicted on crosssectional imaging during radiation planning, which may allow us to reduce the risk of RILD even more ${ }^{[34]}$.

\section{PROPOSED IMAGING ALGORITHM}

We propose that response evaluation should be performed at 3 months after SBRT using multi-phasic CT or MRI. The latter should include DWI sequence and the use of hepatocyte-specific contrast agents should be considered. This should continue 3 monthly in the first year, 3-6 monthly in the second year and 6-12 monthly in the subsequent years depending on the clinical needs. However, the absence of volumetric shrinkage or presence of residual arterial enhancement in the first few months after SBRT should not be deemed tumour relapse unless there are other unequivocal radiological and/or clinical signs of progression. We suggest that early interval imaging should be performed in these cases to confirm disease progression if clinical situation permits.

\section{CONCLUSION}

Early post-SBRT imaging features include hepatic and periportal oedema as well as reactive hyperaemia in the surrounding irradiated liver parenchyma. The latter may mimic disease progression, although there is usually no associated delayed washout observed in these cases. In the subsequent months after SBRT, there is hepatic fibrosis which may be followed by atrophy and capsular retraction. PET imaging is currently not routinely used in HCC, although ${ }^{18}$ F-FDG PET may have a role for extrahepatic staging, particularly in highgrade HCC. ${ }^{99 \mathrm{~m}}$ Tc-SC SPECT can also be considered to assess liver function before and after SBRT.

\section{DECLARATIONS}

\section{Authors' contributions}

Made substantial contributions to conception, design of the study and performed data interpretation: Yip C Made substantial contributions to the design of the study and data interpretation: Hennedige TP, Cook GJR, Goh V

\section{Availability of data and materials}

Not applicable.

Financial support and sponsorship

None.

\section{Conflicts of interest}

All authors declared that there are no conflicts of interest.

\section{Ethical approval and consent to participate}

Not applicable. 


\section{Consent for publication}

Not applicable.

\section{Copyright}

(c) The Author(s) 2020.

\section{REFERENCES}

1. Méndez Romero A, Wunderink W, Hussain SM, De Pooter JA, Heijmen BJM, et al. Stereotactic body radiation therapy for primary and metastatic liver tumors: a single institution phase I-II study. Acta Oncol 2006;45:831-7.

2. Bujold A, Massey CA, Kim JJ, Brierley J, Cho C, et al. Sequential phase I and II trials of stereotactic body radiotherapy for locally advanced hepatocellular carcinoma. J Clin Oncol 2013;31:1631-9.

3. Culleton S, Jiang H, Haddad CR, Kim J, Brierley J, et al. Outcomes following definitive stereotactic body radiotherapy for patients with Child-Pugh B or C hepatocellular carcinoma. Radiother Oncol 2014;111:412-7.

4. Andratschke N, Alheid H, Allgäuer M, Becker G, Blanck O, et al. The SBRT database initiative of the German Society for Radiation Oncology (DEGRO): patterns of care and outcome analysis of stereotactic body radiotherapy (SBRT) for liver oligometastases in 474 patients with 623 metastases. BMC Cancer 2018;18:283.

5. Scorsetti M, Comito T, Clerici E, Franzese C, Tozzi A, et al. Phase II trial on SBRT for unresectable liver metastases: long-term outcome and prognostic factors of survival after 5 years of follow-up. Radiat Oncol 2018;13:234.

6. Sapisochin G, Barry A, Doherty M, Fischer S, Goldaracena N, et al. Stereotactic body radiotherapy vs. TACE or RFA as a bridge to transplant in patients with hepatocellular carcinoma. An intention-to-treat analysis. J Hepatol 2017;67:92-9.

7. Fajardo LF. The pathology of ionizing radiation as defined by morphologic patterns. Acta Oncol 2005;44:13-22.

8. Reed GB, Cox AJ. The human liver after radiation injury. A form of veno-occlusive disease. Am J Pathol 1966;48:597-611.

9. Eisenhauer EA, Therasse P, Bogaerts J, Schwartz LH, Sargent D, et al. New response evaluation criteria in solid tumours: revised RECIST guideline (version 1.1). Eur J Cancer 2009;45:228-47.

10. Bruix J, Sherman M, Llovet JM, Beaugrand M, Lencioni R, et al. Clinical management of hepatocellular carcinoma. Conclusions of the Barcelona-2000 EASL Conference. J Hepatol 2001;35:421-30.

11. Lencioni R, Llovet J. Modified RECIST (mRECIST) assessment for hepatocellular carcinoma. Semin Liver Dis 2010;30:52-60.

12. Vernuccio F, Godfrey D, Meyer M, Williamson H V., Salama JK, et al. Local tumor control and patient outcome using stereotactic body radiation therapy for hepatocellular carcinoma: iRECIST as a potential substitute for traditional criteria. Am J Roentgenol 2019;213:1232-9.

13. Price TR, Perkins SM, Sandrasegaran K, Henderson MA, Maluccio MA, et al. Evaluation of response after stereotactic body radiotherapy for hepatocellular carcinoma. Cancer 2012;118:3191-8.

14. Mannina EM, Cardenes HR, Lasley FD, Goodman B, Zook J, et al. Role of Stereotactic body radiation therapy before orthotopic liver transplantation: retrospective evaluation of pathologic response and outcomes. Int J Radiat Oncol 2017;97:931-8.

15. Takamatsu S, Kozaka K, Kobayashi S, Yoneda N, Yoshida K, et al. Pathology and images of radiation-induced hepatitis: a review article. Jpn J Radiol 2018;36:241-56.

16. Lall C, Bhargava P, Sandrasegaran K, Shanbhogue AK, Ramsinghani N, et al. Three-dimensional conformal radiation therapy in the liver: MRI findings along a time continuum. J Comput Assist Tomogr 2015:39:356-64.

17. Park MJ, Kim SY, Yoon SM, Kim JH, Park SH, et al. Stereotactic body radiotherapy-induced arterial hypervascularity of non-tumorous hepatic parenchyma in patients with hepatocellular carcinoma: potential pitfalls in tumor response evaluation on multiphase computed tomography. PLoS One 2014;9:e90327.

18. Kimura T, Takahashi S, Takahashi I, Nishibuchi I, Doi Y, et al. The time course of dynamic computed tomographic appearance of radiation injury to the cirrhotic liver following stereotactic body radiation therapy for hepatocellular carcinoma. PLoS One 2015;10:e0125231.

19. Ronot M, Asselah T, Paradis V, Michoux N, Dorvillius M, et al. Liver fibrosis in chronic hepatitis C virus infection: Differentiating minimal from intermediate fibrosis with perfusion CT. Radiology 2010;256:135-42.

20. Yu JI, Park HC, Lim DH, Choi Y, Jung SH, et al. The role of diffusion-weighted magnetic resonance imaging in the treatment response evaluation of hepatocellular carcinoma patients treated with radiation therapy. Int J Radiat Oncol 2014;89:814-21.

21. Lo CH, Huang WY, Hsiang CW, Lee MS, Lin CS, et al. Prognostic significance of apparent diffusion coefficient in hepatocellular carcinoma patients treated with stereotactic ablative radiotherapy. Sci Rep 2019;9:14157.

22. Lee SM, Kim HS, Lee S, Lee JW. Emerging role of 18 F-fluorodeoxyglucose positron emission tomography for guiding management of hepatocellular carcinoma. World J Gastroenterol 2019;25:1289-306.

23. Huang WY, Kao CH, Huang WS, Chen CM, Chang LP, et al. 18F-FDG PET and combined 18F-FDG-contrast CT parameters as predictors of tumor control for hepatocellular carcinoma after stereotactic ablative radiotherapy. J Nucl Med 2013;54:1710-6.

24. Lu RC, She B, Gao WT, Ji YH, Xu DD, et al. Positron-emission tomography for hepatocellular carcinoma: Current status and future prospects. World J Gastroenterol 2019;25:4682-95.

25. Talbot JN, Fartoux L, Balogova S, Nataf V, Kerrou K, et al. Detection of hepatocellular carcinoma with PET/CT: A prospective comparison of 18F-Fluorocholine and 18F-FDG in patients with cirrhosis or chronic liver disease. J Nucl Med 2010;51:1699-706.

26. Wallace MC, Sek K, Francis RJ, Samuelson S, Ferguson J, et al. Baseline and post-treatment 18F-Fluorocholine PET/CT predicts outcomes in hepatocellular carcinoma following locoregional therapy. Dig Dis Sci 2020;65:647-57. 
27. Koay EJ, Owen D, Das P. Radiation-induced liver disease and modern radiotherapy. Semin Radiat Oncol 2018;28:321-31.

28. Munoz-Schuffenegger P, Ng S, Dawson LA. Radiation-induced liver toxicity. Semin Radiat Oncol 2017;27:350-7.

29. Zuckerman E, Slobodin G, Sabo E, Yeshurun D, Naschitz JE, Groshar D. Quantitative liver-spleen scan using single photon emission computerized tomography (SPECT) for assessment of hepatic function in cirrhotic patients. J Hepatol 2003;39:326-32.

30. Bowen SR, Chapman TR, Borgman J, Miyaoka RS, Kinahan PE, et al. Measuring total liver function on sulfur colloid SPECT/CT for improved risk stratification and outcome prediction of hepatocellular carcinoma patients. EJNMMI Res 2016;6:57.

31. Kamimura K, Fukukura Y, Yoneyama T, Takumi K, Tateyama A, et al. Quantitative evaluation of liver function with T1 relaxation time index on Gd-EOB-DTPA-enhanced MRI: Comparison with signal intensity-based indices. J Magn Reson Imaging 2014;40:884-9.

32. Haimerl M, Verloh N, Zeman F, Fellner C, Nickel D, et al. Gd-EOB-DTPA-enhanced MRI for evaluation of liver function: Comparison between signal-intensity-based indices and T1 relaxometry. Sci Rep 2017;7:43347.

33. Yoon JH, Lee JM, Kang H, Ahn SJ, Yang H, et al. Quantitative assessment of liver function by using Gadoxetic Acid-enhanced MRI: Hepatocyte uptake ratio. Radiology 2019;290:125-33.

34. Bowen SR, Saini J, Chapman TR, Miyaoka RS, Kinahan PE, et al. Differential hepatic avoidance radiation therapy: proof of concept in hepatocellular carcinoma patients. Radiother Oncol 2015;115:203-10. 